\title{
Epidermal growth factor receptor determination on tumour imprints
}

\author{
D J Hastrich, J M Dunn, P Newcomb, P Hall, N Rooney, S Nicholson, J R Farndon
}

\begin{abstract}
Epidermal growth factor receptor (EGFR) is a sensitive prognostic indicator in women with breast cancer. A new technique for the detection of EGFR on breast cancer imprints is described and evaluated in relation to immunohistochemistry on frozen sections. The standard streptavidin-biotin method using the $\mathrm{R} 1$ antibody on frozen sections was modified for use on tumour imprints. In the first group (group 1 imprints) 43 tumours were assessed by frozen section and imprint staining. The group 1 imprint method had a sensitivity of $50 \%$ and a specificity of $89 \%$ compared with the frozen sections. Further modifications were made for the following 32 tumours (group 2) and this method had a much improved sensitivity of $94 \%$ while specificity was preserved.
\end{abstract}

This method is simple and could be performed in any hospital histopathology department.

(F Clin Pathol 1993;46:166-168)

Epidermal growth factor receptor (EGFR) is a sensitive prognostic indicator in women with breast cancer. ${ }^{12}$ Regardless of axillary nodal status, women with EGFR positive tumours have a reduced disease free and overall survival. ${ }^{1}$ EGFR positivity predicts a poor response to endocrine treatment when used as primary treatment for early breast cancer in the elderly ${ }^{3}$ and when used following relapse after surgery or radiotherapy ${ }^{4}$ in younger women.

Knowledge of EGFR status may help select high risk patients for more aggressive treatment. There is also growing interest in new treatment modalities using "armed" antibodies and immunotoxins to receptors such as EGFR. $^{5}$

EGFR status can be determined by several different methods. The radioligand assay ${ }^{6}$ and immunohistochemistry using the R1 monoclonal $^{7}$ antibody (Amersham, Aylesbury, Buckinghamshire) are the two most commonly used techniques. Both methods are limited in that they can only be performed on fresh tissue, and some tumours are too small to provide enough tissue for both histological examination and receptor studies.

An alternative method for the detection of EGFR has been developed. Immunocyto- chemistry was performed on tumour imprints using the R1 antibody and by modifying the standard streptavidin biotin technique. This paper describes the technique and compares the results of imprint staining with the proved technique of immunohistochemistry on frozen sections.

\section{Methods}

Immunohistochemical staining on frozen sections and on tumour imprints was carried out on the tumours of 70 women undergoing surgery for primary operable breast cancer between May 1991 and February 1992. After appraisal of the early results (group 1 imprints) the method was modified further, attempting to improve the sensitivity (group 2 imprints).

Fresh operative specimens were collected from theatre and were processed immediately in the histopathology department. Imprints were taken by gently laying a glass slide on the cut surface of the tumour. A small sliver of tumour was snap frozen in liquid nitrogen and stored at $-70^{\circ} \mathrm{C}$. All tumours were routinely examined after formalin fixation.

Frozen sections $(7 \mu \mathrm{m})$ were cut and were adhered to polylysine coated slides. The slides were dried at room temperature overnight and stained the next day by a standard streptavidinbiotin technique.

The sections were incubated in a moist chamber with:

(1) primary antibody-R1 antibody diluted 1 in 20 in TRIS-buffered saline (TBS) and 1\% normal rabbit serum for 30 minutes;

(2) link antibody-biotinylated rabbit antimouse (Dako, High Wycombe) diluted 1 in 500 in PBS and 5\% human albumin for 30 minutes;

(3) label-streptavidin-biotin complex horseradish peroxidase (Dako, High Wycombe) for 30 minutes;

(4) chromogen-3,3 diaminobenzidine tetrachloride-DAB $0.6 \mathrm{mg} / \mathrm{ml}$ in TRIS-buffer, $\mathrm{pH}$ $7 \cdot 6$ (Sigma, Poole) for 10 minutes.

Placenta (which has a very high concentration of EGFR) was used as the positive control. The primary antibody was omitted in negative control slides. A negative control was performed for each tumour and a positive control was included in each batch of staining.

IMPRINT IMMUNOCYTOCHEMISTRY

The tumour imprints were air-dried at room temperature overnight, fixed in cold acetone $\left(4^{\circ} \mathrm{C}\right)$ for 10 minutes, and stored at $4^{\circ} \mathrm{C}$. 
Group 1 imprints

The modifications to the standard streptavidin-biotin technique described above for the frozen section immunohistochemistry were:

(1) the concentration of the primary R1 antibody was increased from 1 in 20 to 1 in 10 ;

(2) the label was changed from horseradish peroxidase (HRP) to alkaline phosphatase;

(3) the chromogen was changed from DAB to Fast Red TR.

A cytospin of A431 cells which overexpress EGFR was used as the positive control in each batch. A negative control (primary antibody omitted) was performed for each tumour.

\section{Group 2 imprints}

The method was further modified, incorporating the use of part of the Biogenex StrAviGen Super Sensitive kit (Biodiagnostics, Uptonupon-Severn). This kit has been primarily developed for use on frozen sections. The link antibody provided in the kit was not used, because it was found to give positive staining in the negative control slides in early testing. The streptavidin alkaline phosphatase label (Super Sensitive Stravigen kit) was diluted 1 in 10 and this replaced the standard alkaline phosphatase conjugated streptavidin incubation. In this reagent more alkaline phosphatase is bound to the streptavidin, giving a multiplication effect to the amount of chromogen able to bind to a single receptor. It has been used to enhance immunohistochemical staining in other tissues. $^{8}$

Thirty six tumour imprints were stained by method 2 and the result compared with staining with R1 immunohistochemistry.

Each slide was evaluated independently by two observers (DJH, PN). Staining was scored on the percentage of malignant cells stained as the intensity tended to vary between batches. Staining in both frozen sections and imprints was said to be positive if more than $10 \%$ of the malignant cells were stained. In the imprints the staining sometimes looked cytoplasmic (figure) rather than the classical peripheral membrane staining seen in the frozen sections. This is because one is looking at the membrane binding on the surface of the cell. Slides were graded 0 to +++ , according to the percentage of cells stained: $0 \%$ cells $0,<10 \%$ cells ,$+ 10-75 \%$ cells,$++>75 \%$ cells +++ . Tumours scoring $0 /+$ were said to be negative, those scoring $++1+++$ were judged to be positive.

Imprint taken from the cut surface of the resected specimen and stained with $R 1$ monoclonal antibody to EGF
When benign elements stained, this was noted but did not contribute to the staining score of the malignant cells.

Concordance between the two observers was good. Ten per cent of the total number of slides fell into a borderline group and required joint review before a final decision was made.

Fisher's exact two-tailed test was used for statistical analysis.

\section{Results}

Seventy tumours were examined by frozen section and one of the two methods of imprint staining. Repeat staining was carried out on several sets of imprints, and the intensity and reliability of the EGFR positivity diminished with time. After about a month of being stored at $4^{\circ} \mathrm{C}$, tumours which had previously been strongly positive showed initially weaker, then later, no staining at all. This meant that it was not possible to repeat staining of tumours from the group 1 imprints with the second method for direct comparison.

Forty four tumours were studied by the first method (group 1 imprints). Thirty eight were of invasive ductal nodular sclerosing type. There was one lobular carcinoma, one mucinous carcinoma, and four cases of pure ductal carcinoma in situ (DCIS). The imprints taken from one tumour contained too few cells for adequate assessment, leaving 43 assessable tumours. Of 27 tumours negative for EGFR on frozen section, 24 were also negative on imprint staining; of 16 tumours positive for EGFR on frozen section, eight were positive on the imprints (table). There was good correlation between the two methods but if the frozen section was taken to be the "gold standard", then the specificity of the method in group 1 imprints was $89 \%$ and its sensitivity $50 \%$.

Thirty six tumours were examined by the second imprint method (group 2 imprints). There was a similar distribution of histological types and grades. The table shows the results of the correlation in the 32 tumours where both methods were successfully performed. Although the numbers were smaller, there was an improvement in the sensitivity of method 2 to $94 \%$ while maintaining the specificity at $89 \%$.

\section{Discussion}

The role of EGFR in the clinical management of patients with breast cancer needs further evaluation. Current methods of determining of

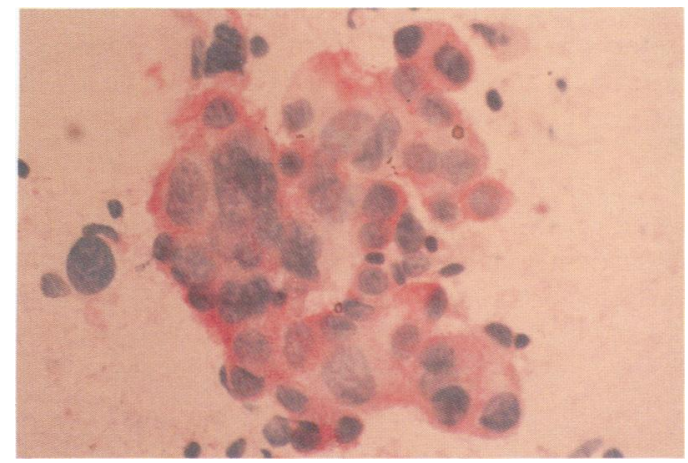

Comparison between staining with the $R 1$ antibody on frozen section and tumour imprints

\begin{tabular}{llll}
\hline & \multicolumn{2}{l}{ Imprints } & \\
\cline { 2 - 4 } Frozen section & Positive & Negative & Total \\
\hline Group $1^{*}$ : Positive & 8 & 8 & 16 \\
Negative & 3 & 24 & 27 \\
Total & 11 & 32 & 43 \\
Group 2t: Positive & 15 & 1 & 16 \\
Negative & 2 & 14 & 16 \\
Total & 17 & 15 & 32 \\
\hline
\end{tabular}

$\star \mathrm{P}=0.01$ (Fisher's exact two-tailed test).

$+p=<0.001$ (Fisher's exact two-tailed test) 
EGFR (the radioligand assay and frozen section immunohistochemistry) have limitations which restrict their use to research centres. The radioligand assay has the benefit of being quantitative but is time consuming, requires facilities for the handling of radioisotopes, and consumes a relatively large amount of fresh tumour. Immunohistochemical staining on frozen sections consumes a smaller piece of fresh tumour but still cannot be used on some small tumours and impalpable localised tumours which need to be formalin fixed before being sectioned.

A reliable antibody for the identification of EGFR on paraffin wax sections would obviate the need for fresh tumour and would permit retrospective study of archival material. The anti-EGFR antiserum 12E (ICRF Oncology Group) has been used successfully in paraffin wax sections of pancreatic tumours but its use has yet to be reported in breast cancers.

Ideally, if EGFR could be identified on fine needle aspiration cytology (FNAC) in the outpatient clinic, information about the basic tumour biology could be taken into account when planning the definitive treatment of each woman. Monoclonal antibodies to steroid receptors ${ }^{10}$ have been used successfully on cytological preparations but there is no reliable method for identifying EGFR on FNAC.

The method of identification of EGFR on tumour imprints described in this paper may be useful and widely applicable until a reliable antibody is found for use on paraffin wax fixed sections. The architecture and orientation of the fresh tumour are not disturbed when the imprint is taken. The imprints can be easily stored or transported under routine laboratory conditions and will keep for about a month. Although specific, the method used with the group 1 imprints was not sensitive enough to be clinically useful. The increased concentration of primary antibody and the increased alkaline phosphatase in the StrAviGen streptavidin complex used in the group 2 imprints improved the sensitivity to $89 \%$ and maintained specificity. With further adaptation, the goal of a simple method for the determination of EGFR on FNAC may be achieved.

1 Nicholson S, Sainsbury JRC, Halcrow P, et al. Epidermal growth factor receptor (EGFr); results of a 6 year followup study in operable breast cancer with emphasis on the node negative subgroup. $B r \mathcal{F}$ Cancer 1991;63:146-50.

2 Toi M, Nakamura T, Mukaida $H$, et al. Relationship between epidermal growth factor receptor status and various prognostic factors in human breast cancer. Cancer 1990;65:1980-4.

3 Nicholson S, Halcrow P, Sainsbury JRC, et al. Epidermal growth factor receptor (EGFr) status associated with failure of primary endocrine therapy in elderly postmenfailure of primary endocrine therapy in elderly postmen-
opausal patients with breast cancer. $B r \quad \mathcal{F}$ Cancer opausal patients
1988;58:810-14

4 Nicholson S, Sainsbury JRC, Halcrow P, et al. Expression of epidermal growth factor receptors associated with lack of response to endocrine therapy in recurrent breast cancer. Lancet 1989;333:182-5.

5 Siegall CB, FitzGerald, DJ, Pastan I. Selective killing of tumour cells using EGF or TGFa-Pseudomonas exotoxin chimeric molecules. Semin Cancer Biol 1990;1:345-50.

6 Carpenter G. Binding assays for epidermal growth factor. Methods Enzymol 1985;109:101-10.

7 Waterfield MD, Mayes EL, Stroobant P, et al. A monoclonal antibody to the human epidermal growth factor receptor. $\Im$ Cell Biochem 1982;20:149-61.

8 Foley JF, Dietrich DR, Swenberg JA, Maronpot RR. Detection and evaluation of proliferating cell nuclear Detection and evaluation of proliferating cell nuclear
antigen (PCNA) in rat tissue by an improved immunohisantigen (PCNA) in rat tissue by an improved immunohis-

9 Lemoine NR, Hughes CM, Barton CM, et al. The epidermal growth factor receptor in human pancreatic cancer. $\mathcal{F}$ Pathol 1992;166:7-12.

10 Skoog L, Humla S, Isaksson S, Tani E. Immunocytochemical analysis of receptors for estrogen and progesterone in fine-needle aspirates from human breast carcinomas. Diagnost Cytopathol 1990;6:95-8. 